\title{
Control of CA3 Output by Feedforward Inhibition Despite Developmental Changes in the Excitation-Inhibition Balance
}

\author{
Christine L. Torborg, ${ }^{1}$ Toshiaki Nakashiba, ${ }^{2}$ Susumu Tonegawa, ${ }^{2}$ and Chris J. McBain ${ }^{1}$ \\ ${ }^{1}$ Program in Developmental Neurobiology, Eunice Kennedy Shriver National Institute of Child Health and Human Development, National Institutes of \\ Health, Bethesda, Maryland, 20892, and ${ }^{2}$ The Picower Institute for Learning and Memory and RIKEN-MIT Center for Neural Circuit Genetics at \\ Massachusetts Institute of Technology, Cambridge, Massachusetts 02139
}

In somatosensory cortex, the relative balance of excitation and inhibition determines how effectively feedforward inhibition enforces the temporal fidelity of action potentials. Within the CA3 region of the hippocampus, glutamatergic mossy fiber (MF) synapses onto CA3 pyramidal cells (PCs) provide strong monosynaptic excitation that exhibit prominent facilitation during repetitive activity. We demonstrate in the juvenile CA3 that MF-driven polysynaptic IPSCs facilitate to maintain a fixed EPSC-IPSC ratio during short-term plasticity. In contrast, in young adult mice this MF-driven polysynaptic inhibitory input can facilitate or depress in response to short trains of activity. Transgenic mice lacking the feedback inhibitory loop continue to exhibit both facilitating and depressing polysynaptic IPSCs, indicating that this robust inhibition is not caused by the secondary engagement of feedback inhibition. Surprisingly, eliminating MF-driven inhibition onto CA3 pyramidal cells by blockade of $\mathrm{GABA}_{\mathrm{A}}$ receptors did not lead to a loss of temporal precision of the first action potential observed after a stimulus but triggered in many cases a long excitatory plateau potential capable of triggering repetitive action potential firing. These observations indicate that, unlike other regions of the brain, the temporal precision of single MF-driven action potentials is dictated primarily by the kinetics of MF EPSPs, not feedforward inhibition. Instead, feedforward inhibition provides a robust regulation of CA3 PC excitability across development to prevent excessive depolarization by the monosynaptic EPSP and multiple action potential firings.

\section{Introduction}

In cortex and CA1 hippocampus, action potential precision is regulated by feedforward inhibition, a circuit configuration in which principal cells and inhibitory interneurons (INTs) are excited simultaneously by common afferents, with INTs in turn inhibiting principal cells with a short delay relative to the monosynaptic excitation. This circuit arrangement dictates the temporal window for action potential initiation (Pouille and Scanziani, 2001; Gabernet et al., 2005; Higley and Contreras, 2006; Luna and Schoppa, 2008). The axons of dentate gyrus granule cells (GCs), the so-called mossy fibers (MFs), provide monosynaptic excitatory synaptic input onto both CA3 pyramidal cells (PCs) and INTs (Acsady et al., 1998), creating the anatomical basis for a robust feedforward inhibitory network. The role played by this extensive inhibitory network in controlling GC-driven action potential timing in CA3 PCs is largely unexplored. Unitary mossy fiber-CA3 pyramidal cell excitatory synaptic events have a rapid rise time, are large in amplitude ( $\sim 60-90 \mathrm{pA})$ (Jonas et al., 1993; Lawrence et al., 2004), and possess marked short term-dependent

Received June 15, 2010; revised Sept. 10, 2010; accepted Sept. 25, 2010.

This study was supported by National Institute of Child Health and Human Development intramural funding to C.J.M., a Pharmacology Research Associate (PRAT) fellowship to C.L.T., and National Institutes of Health Grants R01-MH078821 and P50-MH58880 to S.T. We thank Brian Jeffries for providing technical support and Ken Pelkey for comments on the manuscript.

Correspondence should be addressed to Chris J. McBain, Program in Developmental Neurobiology, Building 35, Room 3C903, Bethesda, MD 20892. E-mail: mcbainc@mail.nih.gov.

DOI:10.1523/JNEUROSCI.3099-10.2010

Copyright $\odot 2010$ the authors $\quad 0270-6474 / 10 / 3015628-10 \$ 15.00 / 0$
(Henze et al., 2002) and frequency-dependent facilitation (Salin et al., 1996), suggesting that MF-driven EPSPs onto CA3 PCs alone may determine the timing of CA3 PC action potential firing (Williams and Johnston, 1991).

In cortical networks, the differential properties of excitatory and inhibitory synaptic short-term plasticity leads to progressive changes in the balance of excitation and inhibition and a degradation of action potential precision (Gabernet et al., 2005). In hippocampus, GCs fire action potentials in $10-40 \mathrm{~Hz}$ bursts (Jung and McNaughton, 1993; Henze et al., 2002), making shortterm plasticity a key component of CA3 circuit function. Thus, to reliably counteract excitation, inhibitory synapses would need to facilitate during repetitive stimulation. However, Mori et al. (2004) observed in slice cultures that IPSPs onto CA3 PCs dominate early in response to a train of presynaptic GC action potentials, with EPSPs dominating later in the train, raising the possibility that the temporal precision of MF-driven action potentials may erode during a train of stimuli. However, this observation contradicts Henze et al. (2002), who demonstrated that INT action potential probability progressively increased during a train of GC action potentials in vivo, suggesting that polysynaptic inhibition onto CA3 PCs facilitates during short-term plasticity. Furthermore, studies of monosynaptic MF to INT connections demonstrate that a subset of INTs receive facilitating MF-driven EPSCs (Toth et al., 2000; Szabadics and Soltesz, 2009), suggesting a role for facilitation within the CA3 feedforward inhibitory network.

Here, we demonstrate in acute hippocampal slices of juvenile rats and mice that MF-driven polysynaptic inhibitory currents 
onto CA3 PCs facilitate; however, in young adult mice MFmediated inhibition either facilitates or depresses, indicating that the short-term temporal dynamics of inhibitory circuit recruitment changes with synaptic maturation. Block of MF-driven inhibition onto CA3 pyramidal cells had minimal impact on the temporal precision of the EPSP-action potential sequence observed after each stimulus but triggered in many cases a long-lasting excitatory plateau potential capable of triggering repetitive action potential firing.

\section{Materials and Methods}

Hippocampal slice preparation. All experiments were conducted in accordance with animal protocols approved by the National Institutes of Health Animal Care and Use Committee. Sixteen- to 20-d-old Sprague Dawley rats (Taconic) or 16- to 20-d-old (juvenile) or 30-to 40-d-old (young adult) mice were deeply anesthetized using isoflurane before decapitation. The brain was removed and placed in cold artificial CSF (ACSF) containing (in $\mathrm{mm}$ ): 50 sucrose, $80 \mathrm{NaCl}, 3.5 \mathrm{KCl}, 1.25$ $\mathrm{NaH}_{2} \mathrm{PO}_{4}, 25 \mathrm{NaHCO}_{3}, 0.5 \mathrm{CaCl}_{2}, 7 \mathrm{MgCl}_{2}, 10$ glucose saturated with $95 \% \mathrm{O}_{2} / 5 \% \mathrm{CO}_{2}, \mathrm{pH}$ 7.4. Transverse hippocampal slices $(300 \mu \mathrm{m})$ were cut on a VT1000S microtome (Leica Microsystems) and placed in a warm $\left(36^{\circ} \mathrm{C}\right.$ ) interface chamber containing the following (in mM): $130 \mathrm{NaCl}$, $3.5 \mathrm{KCl}, 1.25 \mathrm{NaH}_{2} \mathrm{PO}_{4}, 25 \mathrm{NaHCO}_{3}, 0.5 \mathrm{CaCl}_{2}, 5 \mathrm{MgCl}_{2}, 10$ glucose saturated with $95 \% \mathrm{O}_{2} / 5 \% \mathrm{CO}_{2}, \mathrm{pH} 7.4$, until use.

Mice used for these experiments were either wild-type C57BL/6 (National Cancer Institute, Division of Cancer Treatment, Frederick, MD) or "CA3-TeTX" mutants and littermate control mice. CA3-tetanus toxin (TeTX) mice have been described previously (Nakashiba et al., 2008). Briefly, CA3-TeTX mutant mice contain three transgenes. Transgene (Tg) 1 is a Cre transgenic line under control of the transcriptional promoter from the kainate receptor $1(K A-1)$ gene. Tg2 is a tetracycline transactivator transgenic line under control of the calcium/calmodulindependent protein kinase II $\alpha$-subunit ( $\alpha$-CaMKII) promoter and the loxP-Stop-loxP cassette. Tg3-TeTX is a tetanus toxin transgenic line under control of the tetracycline operator. CA3-TeTX mutant mice contain all three transgenes; therefore, in the absence of doxycyline, tetanus toxin is expressed exclusively in CA3 PCs, thus eliminating their ability for downstream transmission and engagement of the CA3 feedback inhibitory circuit. Littermate control mice contain only $\mathrm{Tg} 1$ and $\mathrm{Tg} 3$; therefore, they do not express tetanus toxin and have normal synaptic transmission. For all experiments, CA3-TeTX mice were never fed doxycycline; consequently, tetanus toxin expression in CA3-TeTX mutant mice begins during approximately the third postnatal week following upregulation of the $\alpha$-CaMKII promoter.

Electrophysiology. Slices were placed in a recording chamber and continuously perfused (2-4 ml/min) with ACSF containing the following (in $\mathrm{mm}$ ): $130 \mathrm{NaCl}, 3.5 \mathrm{KCl}, 1.25 \mathrm{NaH}_{2} \mathrm{PO}_{4}, 25 \mathrm{NaHCO}_{3}, 2.5 \mathrm{CaCl}_{2}, 1.5$ $\mathrm{MgCl}_{2}, 10$ glucose saturated with $95 \% \mathrm{O}_{2} / 5 \% \mathrm{CO}_{2}, \mathrm{pH} 7.4$. CA3 pyramidal cells or stratum lucidum interneurons were visually identified using a $40 \times$ objective and infrared differential interference contrast video microsopy (Zeiss Axioskop). Whole-cell patch-clamp recordings were performed at $32-35^{\circ} \mathrm{C}$ using a Multiclamp $700 \mathrm{~A}$ amplifier (Molecular Devices). Recording electrodes $(2.5-4 \mathrm{M} \Omega$ ) pulled from borosilicate glass (World Precision Instruments) were filled with a solution containing the following (in mM):130 Cs-gluconate, $10 \mathrm{HEPES}, 1 \mathrm{EGTA}, 0.1 \mathrm{CaCl}_{2}, 2$ $\mathrm{Na}_{2}$-ATP, $0.3 \mathrm{Na}_{2}$-GTP, $20 \mathrm{KCl}, 5 \mathrm{MgCl}_{2}, 1$ QX314, 20 BAPTA, pH 7.25, with $\mathrm{CsOH}$. Biocytin $(0.2 \%)$ was added to the recording electrode solution to allow post hoc morphological identification of recorded cells. Data acquisition (filtered at $3 \mathrm{kHz}$ and digitized at $10 \mathrm{kHz}$ ) was performed with PClamp 9.2 software (Molecular Devices). Uncompensated series resistance $(8-15 \mathrm{M} \Omega)$ was continuously monitored using a $-5 \mathrm{mV}$ voltage step and experiments in which the series resistance changed by $>15 \%$ were discontinued. Because INT somas and axons are located in stratum lucidum, stimulation of the MF tract also evokes monosynaptic IPSCs. To reduce contamination by monosynaptic IPSCs, we took great care to directly stimulate granule cell somas with an extracellular stimulating electrode placed in the dentate gyrus of hippocampal slices (see Fig. $1 \mathrm{~A}$ ). To identify the optimal stimulus location, the stimulating electrode was systematically moved across the granule cell layer while monitoring the responses of a CA3 pyramidal cell in voltage clamp. Synaptic responses were evoked by a low-intensity stimulation (10-30 $\mu \mathrm{A}$ for $100 \mu \mathrm{s})$ of the granule cell layer using a monopolar glass electrode and a constant current stimulus isolation unit (BAK Electronics). Once a mossy fiber-like EPSC (fast rise and large short-term facilitation) was identified, the stimulus intensity was decreased to $5 \mu \mathrm{A}$ above threshold (10-30 $\mu \mathrm{A})$. EPSCs were recorded at $E_{\text {glutamate }}$ and IPSCs were recorded at $E_{\mathrm{GABA}}$ (where $E$ is reversal potential). Five stimuli at $20 \mathrm{~Hz}$ were delivered once every $10 \mathrm{~s}$.

For current-clamp experiments, recording electrodes were filled with a solution containing the following (in $\mathrm{mM}$ ): $140 \mathrm{KMeSO}_{4}, 8.5 \mathrm{NaCl}, 5$ HEPES, 0.6 EGTA, $4 \mathrm{Mg}_{2}$-ATP, $0.5 \mathrm{Na}_{2}$-GTP, pH 7.25, with KOH. Mossy fiber inputs were first identified under voltage-clamp configuration as described above before switching to current-clamp configuration. To reliably elicit synaptically driven action potentials, cells were biased to close to their action potential threshold $(\sim-45-50 \mathrm{mV})$. All current traces account for a $-6 \mathrm{mV}$ junction potential.

All drugs were obtained from Tocris Bioscience. The following drugs were used: $\left(2 S, 2^{\prime} R, 3^{\prime} R\right)-2-\left(2^{\prime}, 3^{\prime}\right.$-dicarboxycyclopropyl)glycine (DCGIV, $1 \mu \mathrm{M}$ ), DL-2-amino-5-phosphonopentanoic acid (APV, $100 \mu \mathrm{M}), 6$, 7dinitroquinoxaline-2,3-dione (DNQX, $20 \mu \mathrm{M}$ ), SR95531-hydrobromide $(5 \mu \mathrm{M})$.

Data analysis. All data analysis was performed using NeuroMatic (University College London, London, UK) and custom-written software in IgorPro (Wavemetrics). Because spontaneous IPSCs contributed significantly to the inhibitory charge transfer, the mean spontaneous inhibitory charge transfer (taken from an equivalent time period just before simulation) was subtracted from each trace. Measurements of charge transfer were made from the average of 12-30 trials. Total charge transfer was calculated as the area under the curve for a $48 \mathrm{~ms}$ time window beginning $1.5 \mathrm{~ms}$ after the stimulus artifact. To distinguish facilitating synapses from depressing synapses, the $\mathrm{S} 5 / \mathrm{S} 1$ ratio was calculated. Those cells with an S5/S1 ratio $>1$ were considered to be facilitating, whereas those cells with an S5/S1 ratio $<1$ were considered to be depressing. The onset of a synaptic event was the time at which the slope of the current or voltage trace crossed a threshold level $(-30 \mathrm{pA} / \mathrm{ms}$ for EPSCs and +40 $\mathrm{pA} / \mathrm{ms}$ for IPSCs). Latencies were calculated as the time between the stimulus artifact and the onset of the synaptic event or action potential. Only events that occurred within $15 \mathrm{~ms}$ of the stimulus were included in the analysis. The jitter was calculated as the standard deviation of the latency within each cell.

Histological methods. Slices containing biocytin-filled cells were fixed with $4 \%$ paraformaldehyde in $0.1 \mathrm{M}$ phosphate buffer overnight at $4^{\circ} \mathrm{C}$, permeabilized with Triton X-100, and incubated with Alexa Fluor 488conjugated avidin (Invitrogen). Following multiple washes, slices were resectioned to $100 \mu \mathrm{m}$ and mounted on gelatin-coated slides using Mowiol (Calbiochem) mounting medium. Confocal image stacks of stained cells were obtained with a Leica TCS SP2 RS confocal microscope and drawn using Neurolucida.

\section{Results}

The prevalence of MF synapses onto CA3 stratum lucidum INTs suggests that inhibitory interneurons are major contributors to CA3 circuit function, yet little is known about the temporal characteristics of MF-driven inhibitory currents in CA3 PCs. To examine the nature of inhibition onto CA3 PCs driven by activity within the dentate gyrus mossy fiber pathway, we stimulated granule cells within the dentate gyrus of juvenile postnatal day (P) 16-20 rat hippocampal slices and recorded excitatory and inhibitory synaptic currents in CA3 PCs (Fig. 1A,B). EPSCs recorded at the experimentally determined GABA reversal potential $\left(E_{\mathrm{GABA}}=-61 \mathrm{mV}\right)$ were identified as "MF-like" based upon fast rise times $(20-80 \%$ rise time $=0.63 \pm 0.03 \mathrm{~ms}, n=8)$ and a large degree of facilitation in response to a brief train of stimuli (Figs. 1 and 2) (Williams and Johnston, 1991; Toth et al., 2000; Lawrence et al., 2004). After identifying MF-mediated EPSCs, cells were then held at the experimentally determined glutamate 

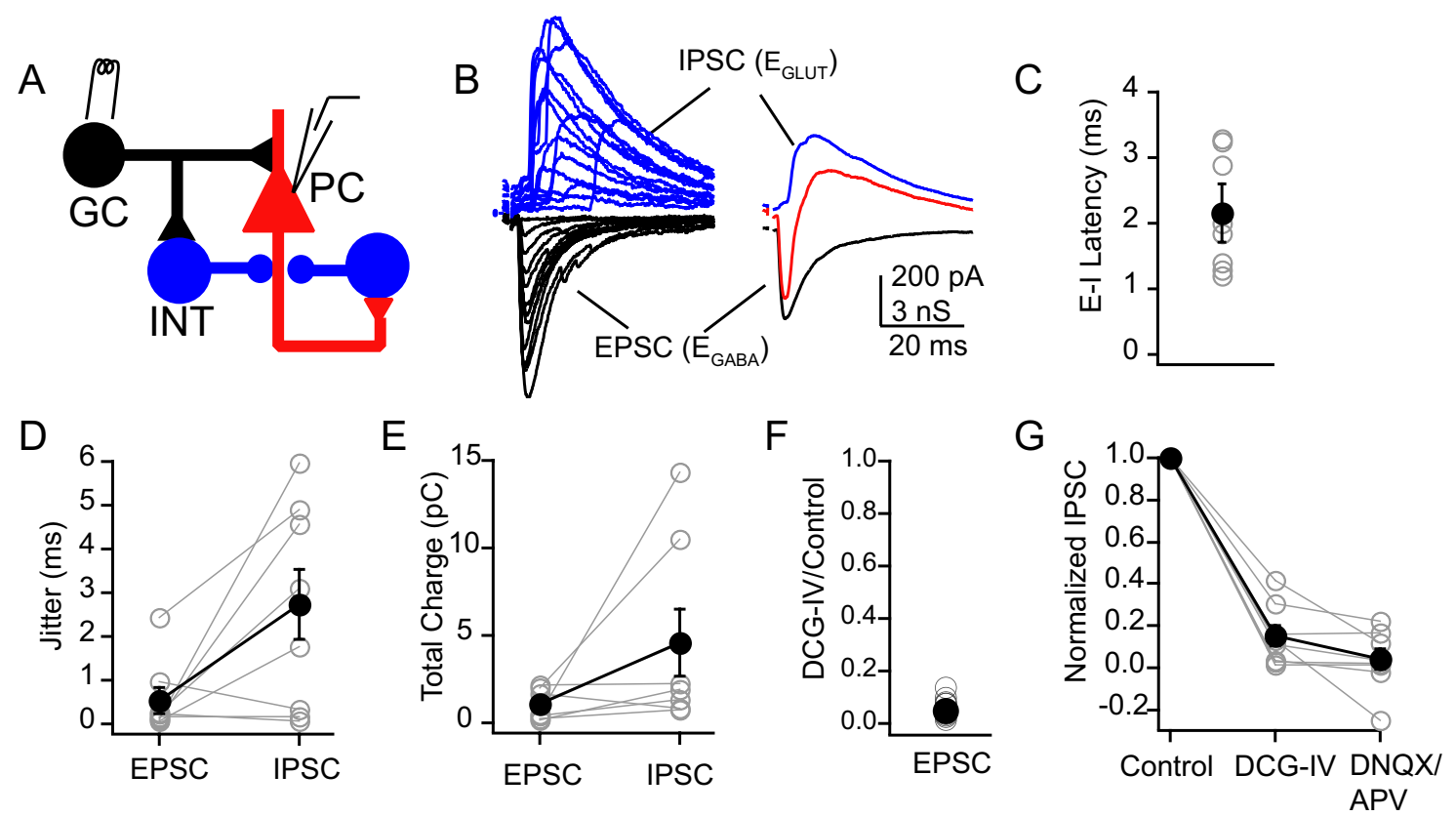

Figure 1. Characteristics of MF-driven polysynaptic inhibition. $A$, Diagram of $(\mathrm{A} 3$ circuit and recording configuration, including granule cell (GC), $C A 3$ pyramidal cell (PC), and inhibitory interneuron (INT). $\boldsymbol{B}$, Example voltage-clamp recording from a $C A 3$ pyramidal cell. Left, Overlay of 12 individual trials of EPSCs recorded at $\mathrm{E}_{\mathrm{GABA}}$ and IPSCs recorded at $\mathrm{E}_{\text {glutamate }}$. Right, Average traces of excitatory currents (black) and inhibitory currents (blue) recorded in a CA3 PC in response to granule cell stimulation. The red trace is the calculated linear sum of the excitatory and inhibitory traces. Because under our recording conditions excitatory and inhibitory currents have equivalent driving forces, the magnitudes of the underlying excitatory and inhibitory conductances have the same ratio as the excitatory and inhibitory currents. Both conductance and current values are included on the scale bar. C, Time difference between the onset of the EPSC and the onset of the IPSC. D, Plot of the jitter (standard deviation of the onset time) for both the EPSC and the IPSC.E, Average charge transfer for the EPSC and IPSC. Open gray circles are individual cells and filled black circles are the mean \pm SEM. $\boldsymbol{F}$, Summary of the charge transfer of the EPSC in the presence of $1 \mu \mathrm{M}$ DCG-IV normalized to the charge transfer under control conditions. G, Graph of the normalized charge transfer of the IPSC in the presence of $1 \mu \mathrm{m}$ DCG-IV or $20 \mu \mathrm{m}$ DNQX and $100 \mu \mathrm{m}$ APV. See also supplemental Fig. S1.

reversal potential $\left(\mathrm{E}_{\text {glutamate }}=10 \mathrm{mV}\right)$ to isolate $\mathrm{MF}$-driven IPSCs. Of 11 CA3 PCs that exhibited MF-mediated EPSCs, eight also received network-driven IPSCs. Analysis of the evoked EPSCs and IPSCs revealed that although EPSC rise times were typically rapid with little variability (jitter) in their onset, individual IPSCs had variable onset latencies. In polysynaptic circuits, each additional synapse acts to increase both the onset latency and the jitter of the onset latency. The monosynaptic EPSC onset was $2.2 \pm 0.4 \mathrm{~ms}$ ( $n=8$ cells) before the onset of the IPSC (Fig. $1 B, C$ ), consistent with previous studies of polysynaptic inhibition (Pouille and Scanziani, 2001; Gabernet et al., 2005). In contrast to the EPSC, the onset of the IPSC was not time locked from trial to trial, such that the onset latency variability (jitter $=2.7 \pm$ $0.8 \mathrm{~ms}$ ) was 5 times greater than monosynaptic EPSC onset latency variability (jitter $=0.5 \pm 0.3 \mathrm{~ms} ; p<0.05)($ Fig. $1 D)$. These data are consistent with MF input driving a monosynaptic EPSC and a polysynaptic IPSC onto the CA3 pyramidal cell population.

The variability in network-driven IPSC timing made consistently defining a peak in the mean IPSC difficult; therefore, we calculated total charge transfer of the mean IPSC (derived from 30 trials), including all inhibitory events evoked by the stimulus regardless of their precise timing. The average excitatory charge transfer was $1.1 \pm$ $0.3 \mathrm{pC}(n=8)$, while the average inhibitory charge transfer associated with MF stimulation was $4.6 \pm 1.9 \mathrm{pC}(n=8)$ (Fig. $1 B-E)$. Although the ratio of the average charge transfer is 0.24 , a more accurate method of determining the ratio of excitation to inhibition ( $E / I$ ratio) is to calculate the E/I ratio within each cell and then take the average of these ratios. Using this method, the average ratio of excitation to inhibition within each cell is $0.57 \pm 0.25(n=8)$.

To confirm that events were indeed MF-mediated postsynaptic currents, the group II metabotropic glutamate receptor antag- onist DCG-IV ( $1 \mu \mathrm{M})$, which selectively depresses release at mossy fiber synapses (Kamiya et al., 1996; Yoshino et al., 1996), was applied at the end of all experiments (Fig. $1 F, G$ and supplemental Fig. S1). EPSCs onto CA3 PCs were reduced to $5 \pm 2 \%$ of control, consistent with them being MF-driven (Fig. $1 F$ ). IPSC charge transfer was also reduced by DCG-IV to $15 \pm 5 \%$ of control. Since DCG-IV does not affect INT-PC synapses (supplemental Fig. S1), the reduction in the IPSC charge transfer in the presence of DCG-IV is entirely due to a decrease in excitatory synaptic transmission at MF-INT synapses. The IPSC charge transfer was not further reduced by inclusion of the glutamatereceptor antagonists DNQX and APV $(4 \pm 5 \%$ of control; $p=$ 0.1 ), indicating that all polysynaptic IPSCs were driven by MF activation and not by direct stimulation of INTs (Fig. 1G).

\section{Mossy fiber EPSCs and polysynaptic IPSCs facilitate to the same degree in juvenile rats}

MF-evoked EPSCs show remarkable facilitation in response to brief trains of activity (Salin et al., 1996; Toth et al., 2000). However, the behavior of MF-driven IPSCs onto CA3 PCs under equivalent conditions has not been previously explored. To examine the relative short-term plasticity within the CA3 circuit, we used a $20 \mathrm{~Hz}$ stimulus delivered to the granule cell layer every $10 \mathrm{~s}$ while recording postsynaptic currents in CA3 PCs. This stimulus frequency was chosen because it closely corresponds to the median frequency of GC firing $(10-40 \mathrm{~Hz}$ ) observed in vivo (Jung and McNaughton, 1993; Henze et al., 2002). Consistent with previous studies (Toth et al., 2000), EPSCs recorded at $E_{\mathrm{GABA}}$ facilitate dramatically (Fig. $2 A, B$ ), with the charge transfer in response to the fifth stimulus (S5) $>7$ times as large as that for the first stimulus $(\mathrm{S} 1)(\mathrm{S} 5 / \mathrm{S} 1=7.6 \pm 2.5 ; n=8)$. Surprisingly, the cor- 

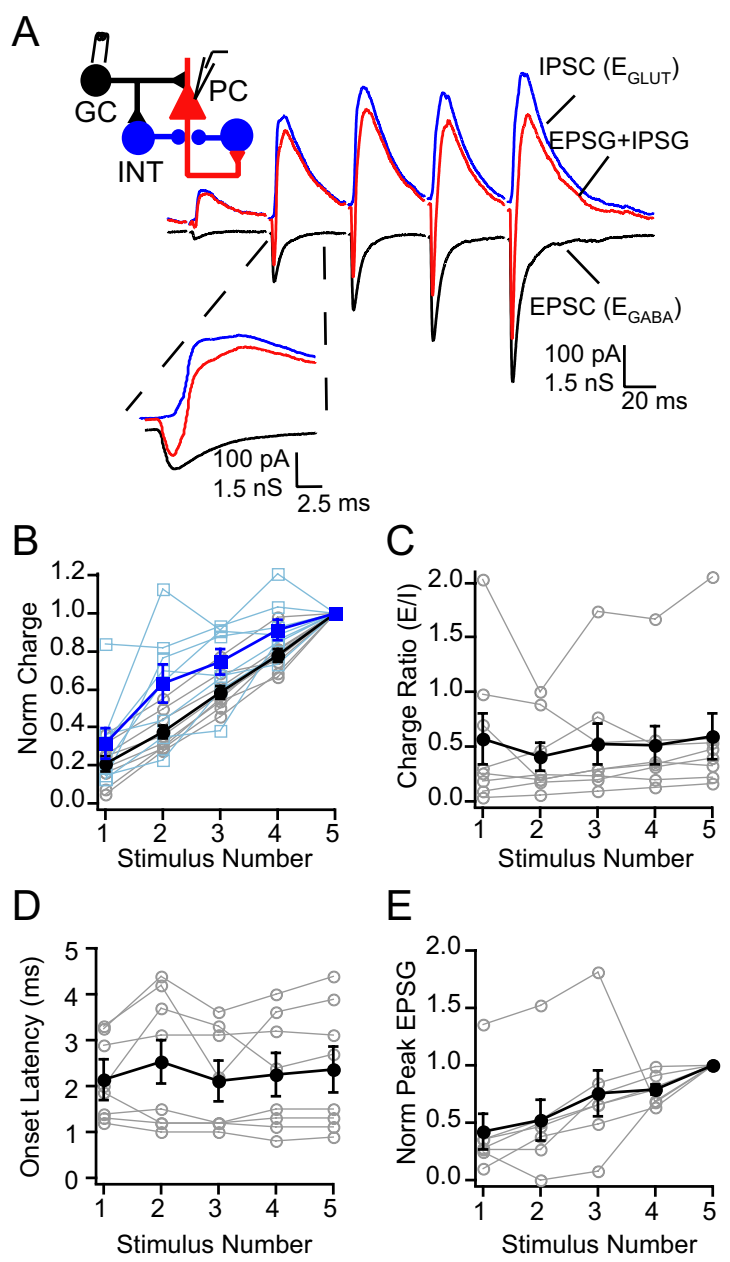

Figure 2. Facilitation of excitation and polysynaptic inhibition in juvenile rats. $\boldsymbol{A}$, Example excitatory (black) and inhibitory (blue) conductances recorded in a CA3 PC in response to granule cell stimulation. For illustrative purposes, the excitatory conductance has been multiplied by -1 . The red trace is the calculated linear sum of the excitatory and inhibitory traces. $\boldsymbol{B}$, Because in a subset of neurons no IPSCS were detected in response to stimulus 1, the excitatory (black circles) and inhibitory (blue squares) charge transfers in response to each stimulus were normalized (Norm) to the response to stimulus 5 and then plotted against stimulus number. $C$, The ratio of excitatory charge transfer to inhibitory charge transfer, E// ratio, was calculated for each cell and plotted as a function of stimulus number (gray open circles). Error bars, SEM. D, Plot of the time difference between the onset of the EPSC and the onset of the IPSC. $\boldsymbol{E}$, The peak amplitude of the excitatory (inward) current was measured from the calculated sum of the EPSC and IPSC (red trace in $\boldsymbol{A}$ ). All amplitudes were normalized to the amplitude in response to stimulus 5. Open symbols, individual cells; closed symbols, average \pm SEM.

responding IPSCs recorded at $E_{\text {glutamate }}$ also facilitate (Fig. $2 A, B$; $\mathrm{S} 5 / \mathrm{S} 1=4.5 \pm 1.0 ; n=8)$, indicating that polysynaptic inhibition is also dominated by facilitating synapses. To determine the balance of excitation and inhibition within each neuron, we calculated the $E / I$ ratio across the train of stimuli. The average $E / I$ ratio for stimulus 1 was $0.57 \pm 0.23(n=8$ cells $)$, indicating that the inhibitory charge transfer was nearly twofold that of the excitatory charge transfer. The $E / I$ ratio for stimulus $5(0.59 \pm 0.21)$ was nearly identical to that of stimulus 1 . In fact, the $E / I$ ratio remained constant over the entire course of the stimulus train (Fig. $2 C$ ) (ANOVA; $p=0.9$ ). Thus, these data demonstrate that the short-term plastic properties of polysynaptic inhibition closely match monosynaptic excitation in juvenile rats.

Our calculation of the E/I ratio does not take into account the temporal dynamics of excitation and inhibition. The $20-80 \%$ rise time of MF-mediated EPSCs was $0.63 \pm 0.03 \mathrm{~ms}$, which is significantly less than the average EPSC-IPSC onset latency difference of $2.2 \pm 0.4 \mathrm{~ms}$ (Figs. $1 C, 2 \mathrm{D}$ ), indicating that inhibition would likely have only a modest effect on the peak of the excitatory conductance. To examine the temporal interaction of excitation and inhibition, we first converted the current trace to a conductance, multiplying the excitatory conductance by -1 for illustrative purposes. Under our recording conditions, excitatory and inhibitory currents have equivalent driving forces; therefore, the magnitudes of the underlying excitatory and inhibitory conductances have the same ratio as the excitatory and inhibitory currents. We then summed the excitatory postsynaptic conductance (EPSG) and inhibitory postsynaptic conductance (IPSG) waveforms (Fig. $2 \mathrm{~A}$, red traces). Immediately after the stimulus, excitation dominated the postsynaptic currents, but this net inward excitatory conductance was quickly replaced by a net inhibitory conductance. Despite this large inhibitory conductance, the peak amplitude of the inward conductance component progressively increased over the course of the stimulus train (S5/S1 $=3.8 \pm$ 1.0) (Fig. 2E). Thus, even though the excitatory and inhibitory charge transfer are balanced, the temporal dynamics of excitatory and inhibitory input continues to permit significant facilitation of the peak of the excitatory conductance.

\section{Do feedforward IPSCs dominate the MF-driven polysynaptic inhibitory circuit?}

The source of facilitation within the polysynaptic inhibitory circuit is unclear. The majority of INT to PC synapses in the hippocampus either depress or do not change strength during repetitive stimulation at $20 \mathrm{~Hz}$ (supplemental Fig. S2), indicating that the short-term plasticity of the monosynaptic INT-PC synapse is unlikely to govern the short-term dynamics of the polysynaptic inhibitory circuit.

If the dominant circuit configuration activated arises through the feedforward inhibitory loop, then the short-term dynamics of excitatory MF inputs onto inhibitory neurons likely contribute to the overall plasticity of polysynaptic MF-driven inhibition. We have previously shown that DCG-IV-sensitive excitatory inputs onto INTs can either depress or facilitate in response to $20 \mathrm{~Hz}$ trains of stimuli (Toth et al., 2000). A similar pattern of shortterm plasticity was observed under our current recording conditions, such that the majority of inputs (17/27) onto INTS showed strong facilitation because of a decrease in failure rate (fraction of trials with no detectable EPSC) during the course of the stimulus train, and 10/27 inputs possessed short-term depression in response to the $20 \mathrm{~Hz}$ stimulus train, which was the result of an increase in the failure rate (Fig. $3 A-C$ ). To confirm that DCG-IVsensitive facilitating EPSCs onto stratum lucidum INTs are monosynaptic and not the result of a MF-driven polysynaptic feedback loop, facilitating DCG-IV-sensitive synapses were first identified while holding the cell at $-60 \mathrm{mV}$ (in the presence of 5 $\mu \mathrm{M}$ SR95531). The holding potential was then changed to +40 $\mathrm{mV}$ and DNQX $(20 \mu \mathrm{M})$ was applied to block all AMPA receptormediated transmission. If glutamate release onto the INT was dependent on a polysynaptic circuit, blockade of AMPA receptors should reduce the depolarization in the intervening CA3 PC to below action potential threshold, thus eliminating the NMDA current in the downstream recorded INT (Salin et al., 1996). However, under these conditions, facilitating NMDA receptormediated currents were still detected in the presence of DNQX, indicating that these DCG-IV-sensitive synapses onto INTs were unequivocally monosynaptic (Fig. 3D). Because monosynaptic facilitating synapses were the dominant type of MF-INT connection observed, it is highly likely that recruitment of 
increasing numbers of INTs during the MF-driven train of activity is what dominates the facilitating polysynaptic inhibition within CA3.

Because numerous subpopulations of stratum lucidum inhibitory interneurons receive mossy fiber afferent input (Toth et al., 2000; Szabadics and Soltesz, 2009), a second possible explanation for this variability is the identity of the postsynaptic interneuron. To identify whether the type of short-term plasticity is correlated with a particular INT subtype, we filled the interneurons with biocytin during recordings and performed post hoc immunohistochemical analysis. The majority of bistratified and radiatum-projecting interneurons (4/5 and 6/8 cells, respectively) received facilitating MF inputs (facilitating synapses were defined as having S5/S1 > 1), while the majority of mossy fiber-associated interneurons received depressing inputs (4/6 cells) (Fig. $3 F$ ). Only one basket cell was recovered, and it received depressing EPSCs. Although all cell types could receive either depressing or facilitating inputs, the trend is for INTs with the bulk of their axon in stratum radiatum to receive facilitating MF synapses. These data suggest that the feedforward inhibition is likely responsible for the facilitation of polysynaptic MFdriven IPSCs; however, it does not rule out the possibility that feedback inhibition also contributes to the inhibitory input onto CA3 PCs.

\section{Do feedback IPSCs contribute to the} facilitation of polysynaptic IPSCs? Another possibility is that MF-driven polysynaptic IPSCs are generated within the feedback inhibitory network. Recurrent CA3 PC axons form an elaborate network of synapses with both other CA3 PCs and INTs. This highly divergent network could form the basis of a strong feedback inhibitory network. Previous studies in vivo have demonstrated that the strong facilitation of the MF-CA3 PC synapse leads to an increase in the probability of CA3 $\mathrm{PC}$ action potentials late in a train of stimuli (Henze et al., 2002). This increased probability of action potentials therefore could also provide the basis for strongly facilitating feedback inhibition.

To distinguish MF-driven feedforward inhibition from any feedback inhibitory component, we switched experimental models to mice, allowing us to use transgenic technology. We used CA3-TeTX mutant mice (Nakashiba et al., 2008), which express tetanus toxin specifically in CA3 PCs. Since tetanus toxin cleaves VAMP2, a protein necessary for vesicular neurotransmitter release, CA3 PCs have reduced neurotransmitter release (supplemental Fig. S3), reducing the possibility of recruiting feedback inhibition without altering feedforward inhibition. In CA3-TeTx mutant mice, tetanus toxin expression does not begin until the third postnatal week

$\mathrm{F}$
B
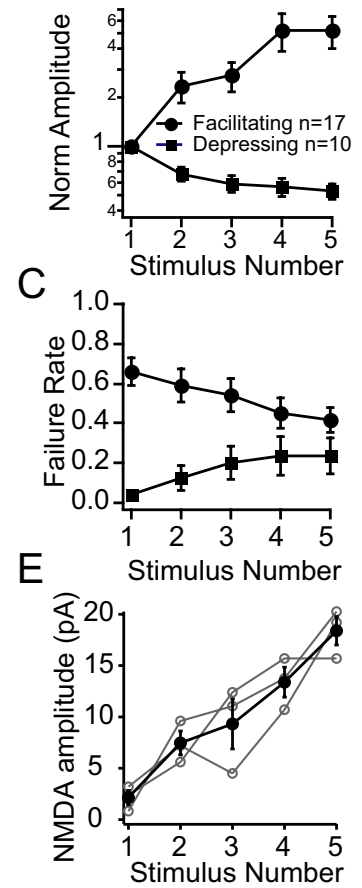
Gabazine/DNQX

$25 \mathrm{~ms}$

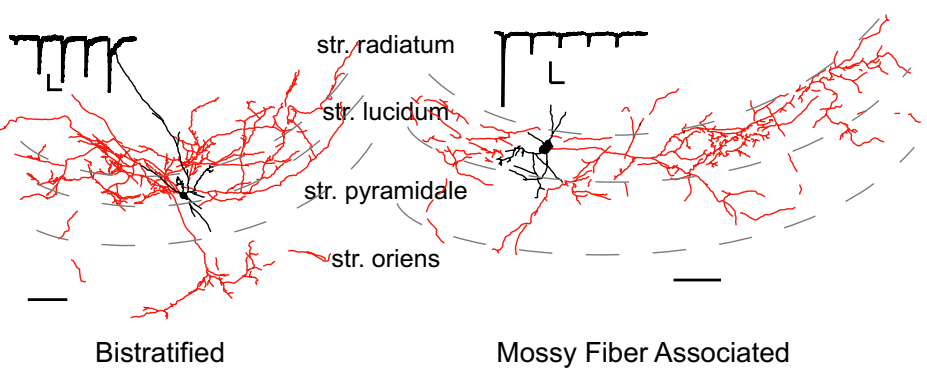

Figure 3. A subset of MF synapses onto INT are facilitating. $A$, Voltage-clamp recordings of stratum lucidum interneurons while extracellularly stimulating the GC layer. EPSCs were sensitive to DCG-IV (green traces, which specifically inhibits MF synapses. See also supplemental Fig. S1. B, Summary of the normalized (Norm) EPSC amplitude on a log scale. EPSC amplitudes were normalized to the amplitude of the first EPSC of the train. EPSCs were considered facilitating if $S 5 / S 1>1$ and depressing if $S 5 / S 1<1$. C, Failure were initially low and then increased. D, Example of a monosynaptic, facilitating, DCG-IV-sensitive input onto an INT. DCG-IVsensitive inputs were found while holding the cell at $-60 \mathrm{mV}$ in the presence of $5 \mu \mathrm{m}$ SR95531. The holding potential was then eceptors. Facilitating NMDAR-mediated currents were still detected in the presence of DNQX, indicating that these inputs onto . 5531.F, To determine whether short-term plasticity was correlated with a particular morphological class of INTs, 列 bistratified cell located in stratum (str.) lucidum that received facilitating excitatory inputs and a mossy fiber-associated cell that received depressing excitatory input.

following upregulation of the $\alpha$-CaMKII promoter (Nakashiba et al., 2008); we therefore performed experiments in mice aged P3040 , where maximal reduction of the feedback loop occurred.

First, to verify that the observed phenomenon of facilitating excitation and inhibition in mice aged P16-20 is similar to that observed in rats, we replicated the key observation of facilitation of polysynaptic MF-driven inhibition in P16-20 C57BL/6 wildtype mice. In mice, MF-mediated polysynaptic IPSCs facilitated during repetitive stimulation and, as observed in rat, the degree of facilitation matched that of EPSCs $(n=5)$ (Fig. $4 A, B)$. In addition, the EPSC-IPSC onset latency differences for stimuli 2-5 are similar between mice and rats ( $p>0.1$ for all stimuli) (Fig. $4 C$, compare to Fig. 2). Finally, the peak EPSG amplitude of the com- 
A
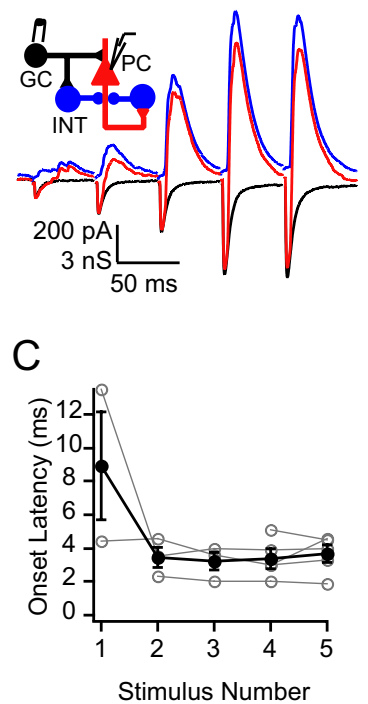

B

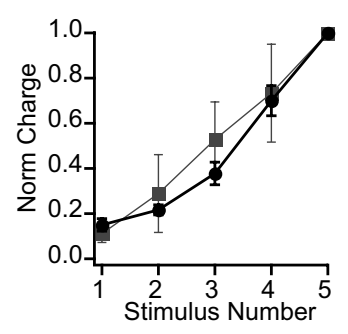

D

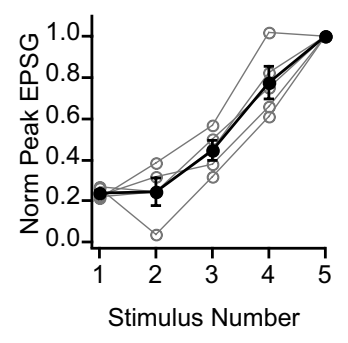

Figure 4. Facilitation of excitation and polysynaptic inhibition in P16-20 mice is similar to that in rats. $\boldsymbol{A}$, Average traces of excitatory currents (black) and inhibitory currents (blue) recorded in a CA3 PC in response to granule cell stimulation. The red trace is the calculated sum of the excitatory and inhibitory conductances. $\boldsymbol{B}$, The mean excitatory (black circles) and inhibitory (gray squares) charge transfer in response to each stimulus was normalized to the response to stimulus 5. Error bars, SEM. C, Plot of the time difference between the onset of the EPSC and the onset of the IPSC. Missing data points are stimuli in which no polysynaptic IPSC was detected. D, The peak amplitude of the excitatory (inward) conductance was measured from the calculated sum of the EPSG and IPSG (red trace in $\boldsymbol{A}$ ). All amplitudes were normalized to the amplitude in response to stimulus 5. Open symbols, Individual cells; closed symbols, average \pm SEM.

bined EPSG-IPSG facilitated fourfold during the stimulus train $(\mathrm{S} 5 / \mathrm{S} 1=4.2 \pm 0.2)($ Fig. $4 D)$, a value similar to the peak EPSG facilitation observed in rats $(\mathrm{S} 5 / \mathrm{S} 1=3.8 \pm 1.0)$ (Fig. $2 E$ ). Thus, the short-term dynamics of polysynaptic MF-mediated inhibition is similar in both juvenile mice and rats.

In P30-40 mice, MF-driven monosynaptic EPSCs onto CA3 PCs continue to facilitate nearly fourfold ( $55 / \mathrm{S} 1=3.6 \pm 0.7 ; n=$ 6) (Fig. $5 A, B$ ) during a $20 \mathrm{~Hz}$ train of five stimuli, similar to the results observed in juvenile animals. Surprisingly, in these recordings the average MF-driven polysynaptic IPSC dataset did not change during a train of stimuli $(\mathrm{S} 5 / \mathrm{S} 1=0.7 \pm 0.4 ; n=6)$ (Fig. $5 B$ ). However, analysis of each individual recording revealed MF-driven IPSCs facilitated in two of six CA3 PCs (S1 = 4.2 \pm $0.2 \mathrm{pC}, \mathrm{S} 5=10.3 \pm 1.7 \mathrm{pC} ; n=2$ ) (Fig. $5 \mathrm{~A}, \mathrm{C}$ ) depressed in two of six CA3 PCs $(\mathrm{S} 1=27.7 \pm 4.6 \mathrm{pC}, \mathrm{S} 5=11.7 \pm 1.9 \mathrm{pC} ; n=2)$, and in the remaining two cells MF-driven IPSCs showed mixed depression followed by facilitation late in the train, resulting in no net change between $\mathrm{S} 1$ and $\mathrm{S} 5(\mathrm{~S} 1=23.9 \pm 0.4 \mathrm{pC}, \mathrm{S} 5=22.8 \pm$ $0.3 \mathrm{pC} ; n=2$ ) (Fig. $5 B, C$ ). Of interest, in those connections that showed depression, the charge transfer associated with inhibition always exceeded the corresponding EPSC charge, ensuring that robust inhibition dominated throughout the stimulus train (Fig. 5D). In fact, near the end of the train of stimuli, the magnitude of the inhibition in cells receiving depressing MF-driven IPSCs was similar to the magnitude of the inhibition in cells receiving facilitating MF-driven IPSCs (Fig. 5C).

To understand better how this shift in the balance of excitation and inhibition during the course of a train of stimuli affects the facilitation of excitatory currents, we converted the currents to conductances, calculated the sum of the EPSG and IPSG, and measured the peak amplitude of the excitatory conductance on

the combined trace (Fig. 5E). If MF-driven polysynaptic inhibition influences the peak excitatory conductance, then the change in the peak conductance should be greater in cells with depressing IPSCs than in cells with facilitating or mixed IPSCs. Surprisingly, there was no correlation between the degree of facilitation of the peak excitatory conductance and the amount of change of the $E / I$ ratio (Fig. 5D-F). Cells with depressing IPSCs, which exhibited a ninefold change in the E/I ratio, had only a twofold change of the peak excitatory conductance $(\mathrm{S} 5 / \mathrm{S} 1=2.3 \pm 0.5)($ Fig. $5 E, F)$. Cells with mixed IPSCs also exhibited a twofold change of the peak excitatory conductance $(\mathrm{S} 5 / \mathrm{S} 1=1.9 \pm 0.7 ; n=2)$ even though they had only a threefold change of the E/I ratio, indicating that the amount of inhibition has only a modest impact on the excitatory conductance peak amplitude. This most likely reflects the rapid rise time of the EPSG, allowing it to reach its peak amplitude before the IPSG onset, which was $\sim 2 \mathrm{~ms}$ after the onset of the EPSG (Fig. 5G).

This unexpected heterogeneity of inhibitory short-term plasticity in young adult mice could be due to heterogeneity within the feedforward network, or it could be due to recruitment of the feedback inhibitory network. To examine whether feedback inhibition plays a role in the short-term plasticity of polysynaptic inhibition, we assayed short-term plasticity in CA3-TeTX mutant mice, which lack the feedback inhibitory loop. In CA3-TeTX mutant mice, MF-driven IPSCs were observed to facilitate in response to short trains of stimuli in four of seven CA3 PCs ( $\mathrm{S} 1=$ $3.6 \pm 1.8 \mathrm{pC}, \mathrm{S} 5=7.8 \pm 2.8 \mathrm{pC})$, but depressed in the remaining three cells $(\mathrm{S} 1=17.2 \pm 1.3 \mathrm{pC}, \mathrm{S} 5=7.1 \pm 2.0 \mathrm{pC})($ Fig. $5 H-J)$. This suggests that, as in control littermate mice, both types of plasticity can be expressed within the feedforward inhibitory circuit and, importantly, that feedback inhibition does not contribute significantly to MF-driven short-term plasticity in CA3 PCs. Interestingly, even in CA3-TeTX mutant mice we observed IPSCs with multiple peaks occurring at long latencies (Fig. $5 \mathrm{H}$, right), indicating that long latency IPSCs can be generated completely within the feedforward inhibitory circuits.

P30-40 control and CA3-TeTX mice did not differ in any of the other parameters we measured. MF-driven EPSCs onto CA3 pyramidal cells facilitated to the same degree in control $(\mathrm{S} 5 / \mathrm{S} 1=$ $3.6 \pm 0.7, n=6)$ and CA3-TeTX (S5/S1 = 5.3 $\pm 0.9, n=7 ; p>$ 0.1 ) mice (Fig. $5 A, B, H, I$ ), indicating that the feedback circuit does not influence the facilitation of MF-driven EPSCs (Kwon and Castillo, 2008). No differences were observed between controls and mutants in the peak amplitude of the combined EPSGIPSG trace (depressing S5/S1 = $2.1 \pm 0.6$; facilitating S5/S1 = $2.1 \pm 1.6 ; p>0.05$ ) (Fig. $5 L$ ), and there was no correlation between the degree of facilitation of the peak excitatory conductance and the amount of change of the $E / I$ ratio (Fig. $5 K-M$ ). Finally, the EPSC-IPSC onset latency difference was not significantly different between control and CA3-TeTX mice (Fig. $5 \mathrm{~N}$ ). We found no detectable difference between control and CA3TeTX mutant mice, confirming that the mixture of depressing and facilitating CA3 polysynaptic inhibition observed in adult mice is entirely due to the heterogeneity of short-term plasticity of synapses within the feedforward inhibitory circuit rather than differential recruitment of the trisynaptic feedback circuit.

GABAergic inhibition does not alter the timing of MF-driven action potentials in CA3 PCs but acts to reduce the probability of bursts of action potentials

Previous studies in cortex and CA1 hippocampus have demonstrated that feedforward inhibition determines spike timing by providing a narrow time window for the temporal integration of 
EPSPs (Pouille and Scanziani, 2001; Luna and Schoppa, 2008). In these studies, blockade of inhibition reduced the temporal precision of action potentials by increasing the latency and jitter (standard deviation of the latency) of action potentials. Furthermore, in somatosensory cortex, Gabernet et al. (2005) demonstrated that although both EPSCs and feedforward IPSCs depress during repetitive stimulation, the magnitude of that depression is greater for IPSCs than for EPSCs. This change in the balance of excitation and inhibition was correlated with an increase in action potential jitter, indicating that subtle changes in the balance of excitation and inhibition during trains of stimuli can lead to reduced temporal precision of action potentials.

Here, using young adult mice (P3040 ), we tested the role of feedforward inhibition during short trains of MF-driven action potentials in CA3 PCs. Using the same stimulus protocol as in the previous experiments, we stimulated granule cells and recorded postsynaptic potentials and action potentials in CA3 PCs in currentclamp configuration. The chloride reversal potential of the intracellular solution was set at $-75 \mathrm{mV}$, which is the physiologically measured chloride reversal potential of CA3 PCs (Banke and McBain, 2006). The low-intensity stimulus protocol that we used was unable to elicit action potentials from CA3 PCs at their resting potential $(-70 \pm 2 \mathrm{mV}$; therefore, cells were biased close to action potential threshold ( -45 to $-50 \mathrm{mV})$. At this potential, MF stimulation evoked single action potentials with a short latency $(4.7 \pm 0.6 \mathrm{~ms})$ and low jitter $(0.76 \pm 0.27$ $\mathrm{ms} ; n=7)$. The probability of action potentials in response to the first stimulus in a train of stimuli was only $0.18 \pm 0.1$, but by the fifth stimulus in the train it more than doubled to $0.4 \pm 0.1(n=7)$. On trials that did not elicit action potentials, subthreshold postsynaptic potentials consisted of a short latency EPSP that was quickly shunted by a slightly longer latency IPSP (Fig. 6A, $B$ ), indicating that MF stimulation did indeed recruit feedforward inhibition.

To determine whether GABAergic inhibition plays a role in establishing the timing of MF-evoked action potentials, we bath applied the GABA receptor antagonist SR95531 $(5 \mu \mathrm{M})$. The elimination of the IPSP by SR95531 (Fig. 5A) had unexpected effects on the probability and timing of action potentials. In three of seven CA3 PCs, MF stimulation evoked plateau potentials on which action potentials were superimposed (Fig. 6A,B). These plateau potentials occurred in $40 \pm 10 \%$ of trials

\section{P30-40 Control}
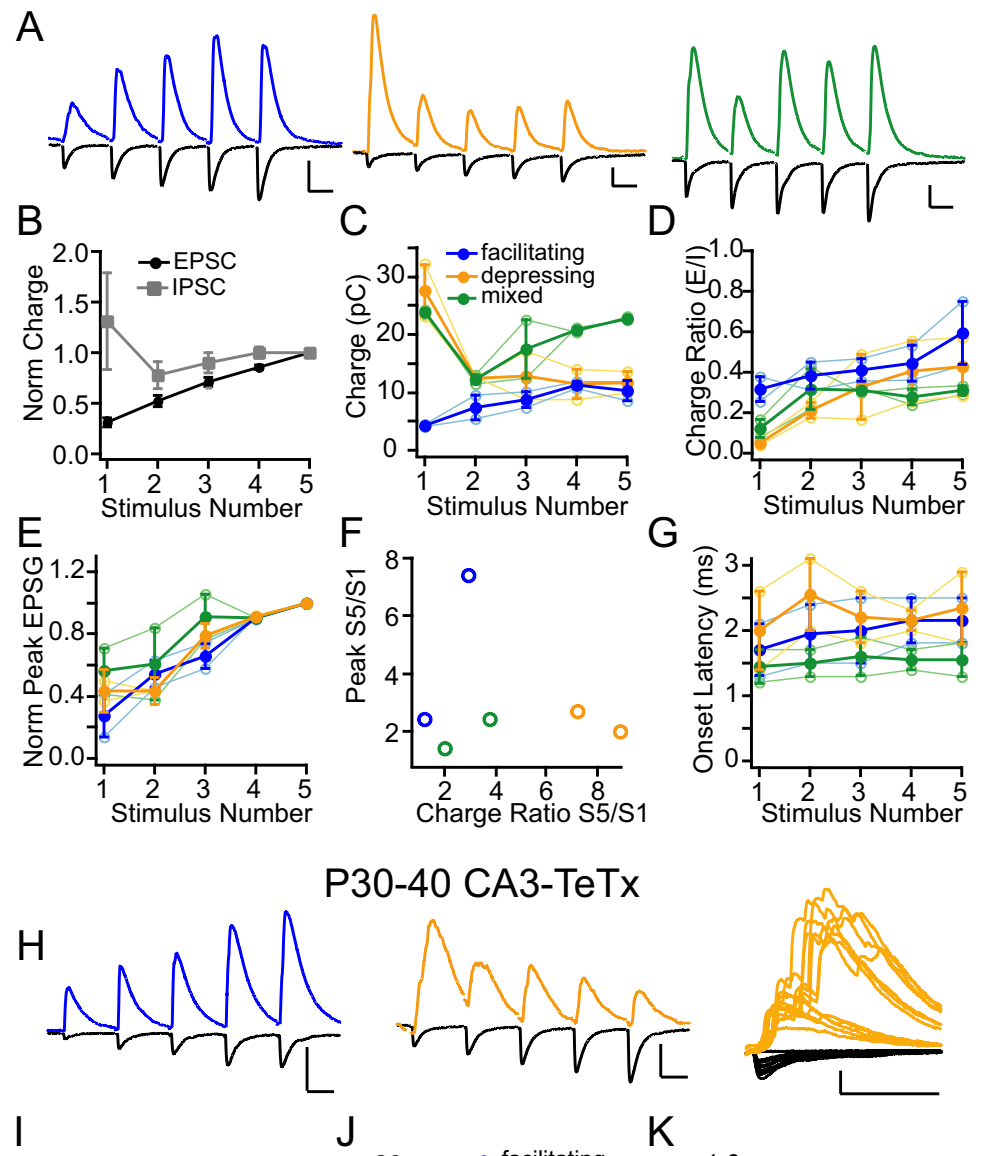

P30-40 CA3-TeTx

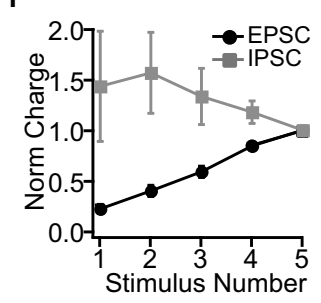

L
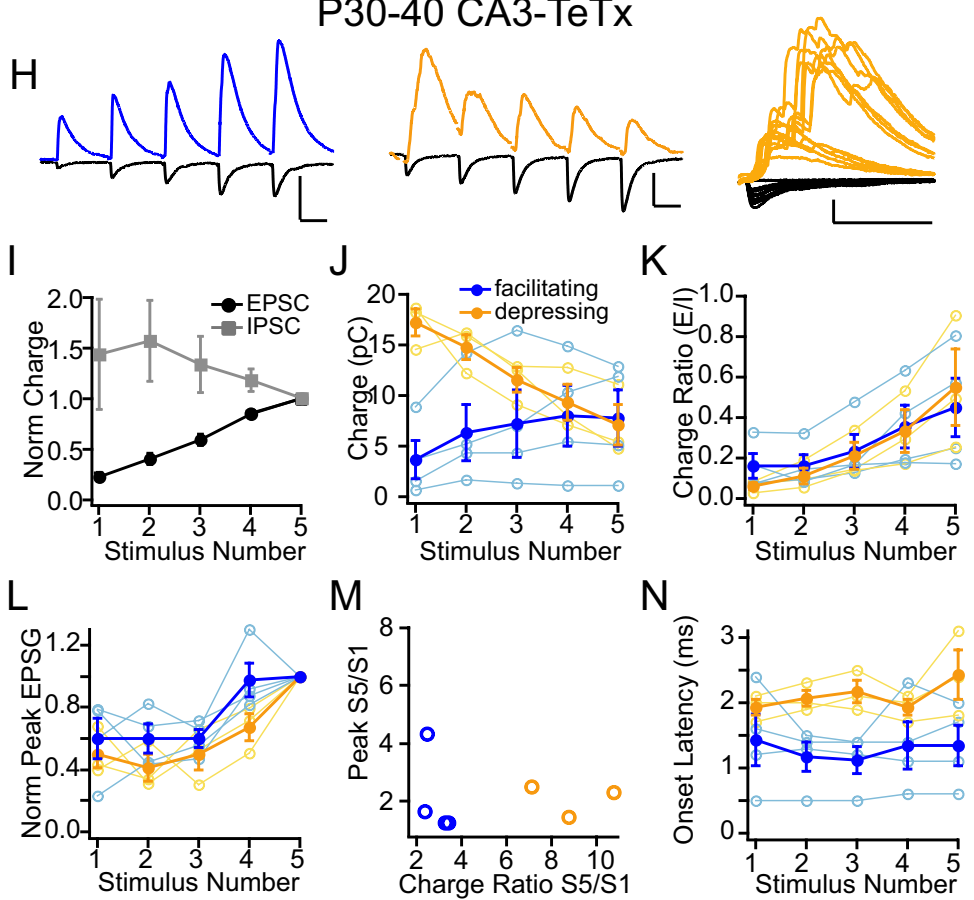

$\mathrm{N}$

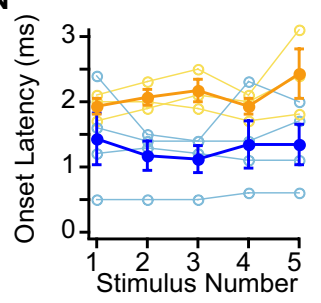

Figure 5. P30-40 control and CA3-TeTX mice exhibit both facilitating and depressing MF-driven polysynaptic IPSCS. P30 - 40 littermate control $(\boldsymbol{A}-\boldsymbol{G})$ and CA3-TeTX $(\boldsymbol{H}-\boldsymbol{N})$ mice exhibited both facilitating polysynaptic IPSCs. $\boldsymbol{A}$, Example average traces of facilitating (left, blue trace), depressing (middle, orange trace), and mixed (right, green trace) IPSCS in P30 - 40 control mice. Black traces are excitatory currents. Scale bar, 250 pA, 25 ms. $\boldsymbol{H}$, Example average traces of facilitating (left, blue trace) and depressing (middle, orange trace) in CA3-TeTX mice. Right, Twelve individual trials from S1 of the same cell as middle. Black traces are excitatory currents. Scale bar, 200 pA, 25 ms. B, I, Plot of the average excitatory (black circles) and inhibitory (gray squares) charge transfer normalized (Norm) to the charge transfer at stimulus 5. C, J, Inhibitory charge transfer was plotted as a function of stimulus number. Cells with facilitating IPSCs are blue, depressing IPSC s are orange, and mixed facilitating and depressing IPSCs are green. $\boldsymbol{D}, \boldsymbol{K}$, The EPSC-IPSC charge transfer ratio increased during the course of the stimulus train for cells with all types of short-term plasticity. $E, L$, The peak amplitude of the excitatory conductance was measured from the calculated sum of the EPSG and IPSG and plotted as a function of stimulus number and normalized to pulse number 5. $\boldsymbol{F}, \boldsymbol{M}$, The peak amplitude the excitatory conductance (from $\boldsymbol{E}, \boldsymbol{L}$ ) was plotted against the charge ratio (from $\boldsymbol{D}, \boldsymbol{K}$ ) for each cell. $\mathbf{G}, \boldsymbol{N}$, Summary of the EPSC-IPSC onset latency difference. Open symbols, Individual cells; closed symbols, average \pm SEM. See also supplemental Fig. S3. 

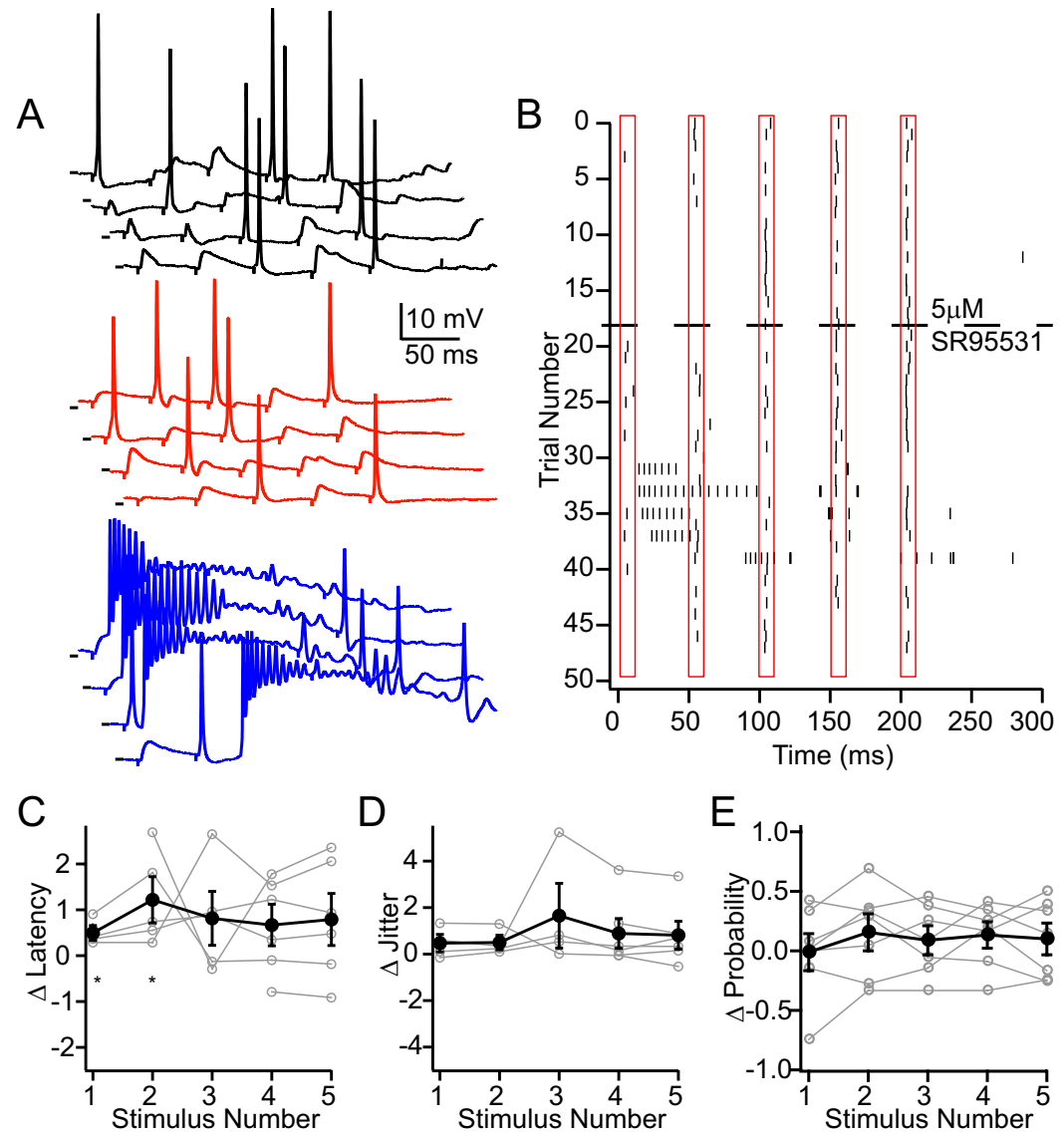

Figure 6. Blockade of $\mathrm{GABA}_{\mathrm{A}}$ receptors increases cell excitability but does not alter temporal precision of single action potentials. $A$, Example current-clamp recording from a CA3 PC that exhibited plateau potentials in the presence of SR95531. Current was injected to bias the resting potential close to action potential threshold. Traces shown are single trials under either control conditions (top, black) or in the presence of SR95531 (middle, red; bottom, blue). In the presence of SR95531, MF stimulation elicited plateau potentials in a subset of trials (bottom traces, red). Scale bar, $3 \mathrm{mV}$ (top traces) or $10 \mathrm{mV}$ (bottom trace). $\boldsymbol{B}$, Raster plot of action potentials for the same cell as that shown in $A$. Five stimuli were delivered every $50 \mathrm{~ms}$ starting at time $0 \mathrm{~ms}$. SR95531 was bath applied at trial 18. C, The change in action potential latency after bath application of SR95531 ( $\Delta$ latency $=$ SR95531 latency - control latency) was plotted as a function of stimulus number. Asterisks denote $p>0.05$. $\boldsymbol{D}$, The change in action potential jitter after bath application of SR95531 ( $\Delta$ jitter $=$ SR95531 jitter - control jitter) was plotted as a function of stimulus number. $\boldsymbol{E}$, The change in action potential probability after bath application of SR95531 ( $\Delta$ probability $=$ SR95531 probability control probability) was plotted as a function of stimulus number. These statistics only include trials on which single action potentials were elicited. Trials exhibiting plateau potentials were not included in the analysis.

and were initiated in response to any of the five stimuli (Fig. $6 A, B)$. If trials that contained plateau potentials are excluded from analysis, the elimination of IPSPs had only a minimal effect on the timing and probability of single action potentials (Fig. $6 C-E)$. The latency of action potentials in response to the first and second stimuli in the train increased slightly in SR95531 ( $\Delta$ latency $\mathrm{S} 1=0.49 \pm 0.16 \mathrm{~ms} ; \mathrm{S} 2=1.2 \pm 0.5 \mathrm{~ms} ; p \leq 0.05$ paired $t$ test) but did not change for the later stimuli. The jitter of MFdriven action potentials was unaltered in SR95531 during the entire train of stimuli (Fig. 6D). Interestingly, SR95531 also did not alter the probability of action potentials on trials in which plateau potentials were not elicited (Fig. 6E). Together, these data indicate that, unlike CA1 hippocampus and cortex, feedforward IPSPs do not influence the timing of action potentials in CA3 PCs. Rather, feedforward IPSPs prevent CA3 PCs from prolonged depolarization and repetitive firing in response to MF-driven EPSPs. In short the role of inhibition in CA3 PC is to stabilize cell excitability and not spike timing.

\section{Discussion}

By influencing the amplitude and kinetics of EPSPs, feedforward inhibition limits the temporal summation of EPSPs and reduces both action potential jitter and number in cortical and CA1 hippocampal PCs (Pouille and Scanziani, 2001; Gabernet et al., 2005; Luna and Schoppa, 2008). In somatosensory cortex, both monosynaptic excitation and disynaptic inhibition onto principal cells undergo short-term synaptic depression during repetitive stimulation; however, feedforward inhibition depresses to a larger degree, altering the balance of excitation and inhibition. This reduction of inhibition relative to excitation leads to a progressively longer temporal window for EPSP summation and an increase in action potential jitter, underscoring the effectiveness of feedforward inhibition in controlling spike timing (Gabernet et al., 2005).

In contrast, in the developing hippocampus we found that MF-driven feedforward inhibition onto CA3 PCs facilitates to nearly the same degree as MF-driven excitation, resulting in balanced excitation and inhibition throughout the train of stimuli. Because of the rapid rise of the EPSC, the excitatory current peak is maximal before the onset of the inhibitory current just $2 \mathrm{~ms}$ later, allowing for the facilitation of the peak excitatory current during repetitive stimulation before the inhibitory current dominates. This arrangement suggests that polysynaptic MF-driven inhibition allows CA3 PC action potential probability to increase during the train of stimuli without a loss of temporal precision. Surprisingly, however, the short-term plasticity of polysynaptic MF-driven inhibition changed during development, such that by P30 - 40 both facilitating and depressing MF-driven polysynaptic IPSCs were evident. Although there was a shift in the balance of excitation and inhibition during a train of stimuli, the addition of depressing IPSCs represented a net increase in the amount of inhibition for stimuli early in a train rather than a decrease in inhibition near the end of the train (Fig. 5C). Importantly, this change in the balance of excitation and inhibition did not alter the timing of action potentials during a train of stimuli, forcing us to rethink the hypothesis that the balance of excitation and inhibition alone is important for maintaining action potential timing during repetitive stimulation. In fact, unlike cortex and CA1 hippocampus, MF-driven feedforward inhibition is not the sole determinant of the temporal precision of single action potentials in CA3 hippocampus. Rather, the role of feedforward inhibition is primarily to control the CA3 PC excitability by preventing plateau potentials and action potential bursts.

The feedforward inhibitory circuit is responsible for inhibition along the apical somatodendritic axis of CA3 PCs. In cortex and hippocampal CA1, feedforward inhibition is mediated al- 
most exclusively by perisomatic, fast-spiking basket cells (Pouille and Scanziani, 2001; Gabernet et al., 2005; Glickfeld and Scanziani, 2006; Daw et al., 2007; Glickfeld et al., 2008). In these circuits, feedforward excitatory synaptic strength onto fast-spiking basket cells either does not change or depresses during repetitive stimulation, and monosynaptic connections between fast-spiking basket cells and principal cells depress during repetitive stimulation, resulting in an overall depression of the feedforward inhibitory drive onto principal cells (Gabernet et al., 2005; Glickfeld and Scanziani, 2006). In hippocampal CA3, the role of fast-spiking basket cells in feedforward inhibition is unclear. In paired recordings between MF boutons and INTs, MF synapses onto fastspiking basket cells are infrequent and exhibit an extremely low release probability with mild facilitation (Szabadics and Soltesz, 2009), suggesting that they could account in part for the facilitation of MF-driven feedforward inhibition observed here. Our data suggest that dendrite-projecting INTs, including radiatumprojecting and bistratified INTs, are more likely to be the dominant participants in feedforward inhibition in CA3 because they receive facilitating MF-driven EPSCs capable of triggering action potential firing (Lawrence et al. 2004). Here and in other studies from our lab (Toth and McBain, 1998; Toth et al. 2000; Pelkey et al., 2005), dendritic targeting interneurons are encountered far in excess of perisomatic targeting interneurons in stratum lucidum.

The emergence of depressing MF-driven feedforward inhibition in young adult mice suggests that additional classes of INTs may be recruited into the circuit during development. Although the majority of MF-associated INTs received depressing MFdriven EPSCs (Fig. 3), they are unlikely to mediate depressing feedforward inhibition, since they release neurotransmitter only at high $(>25 \mathrm{~Hz}$ ) firing frequencies (Losonczy et al., 2004). Regular spiking, cholecystokinin (CCK)-positive basket cells also receive depressing MF-driven EPSCs (Szabadics and Soltesz, 2009). However, in CA1 these cells are involved exclusively in feedback inhibition (Glickfeld and Scanziani, 2006; Glickfeld et al., 2008). Therefore, if CCK basket cells are involved in feedforward inhibition in the CA3 region, it would represent a novel role for these cells within inhibitory circuits.

By engaging different classes of INTs that form synapses along the entire somatodendritic region of principal cells, feedforward inhibition will influence the integration of virtually all types of inputs that CA3 PCs receive. Because of their perisomatic location, IPSPs from basket cell synapses exert significant control over somatic voltage, altering the amplitude and kinetics of somatic EPSPs and the generation of sodium-dependent action potentials (Miles et al., 1996; Pouille and Scanziani, 2001). In contrast, IPSPs from dendritic-projecting INTs alter integration of synaptic inputs within the dendrites and suppress dendritic calcium spikes (Miles et al., 1996; Pouille and Scanziani, 2001) but have limited control over somatic action potential generation (Miles et al., 1996; Pouille and Scanziani, 2001). Whether the dendritic location of radiatum-projecting and bistratified INT inputs hinders their ability to modulate EPSPs from MF synapses, which are located near the soma of CA3 PCs, remains to be tested. However, we predict that the likely role of these dendritic IPSPs is to modify the integration of EPSPs from associational/commissural (A/C) inputs, which form synapses along the length of the dendrites, and entorhinal inputs, which form synapses in the distal dendrites of CA3 PCs at the time when mossy fiber input to CA3 is maximal. In this manner, the primary purpose of MFdriven feedforward inhibition may be to lessen CA3 PC responsiveness to $\mathrm{A} / \mathrm{C}$ and entorhinal cortex inputs, allowing for the specific entrainment of CA3 by MF input.
Our current-clamp recordings illustrate that MF-EPSPdriven action potentials are temporally precise, exhibiting a short latency and narrow jitter. The narrow window for excitatory synaptic-driven action potential generation results from two main properties of the circuit. First, MF-driven EPSPs display a characteristic short latency and have a rapid rise time and a large unitary synaptic conductance. Second, these large facilitating EPSPs are rapidly shunted by the large IPSP to generate a narrow and relatively fixed time window for depolarization (Fig. 6). Importantly our data demonstrate that although feedforward inhibition is capable of rapidly terminating the excitatory synaptic input, it does not appear to govern the temporal precision of single MF-driven action potentials in CA3 PCs. Instead, the temporal precision of action potentials is likely governed by the kinetics of the EPSP itself and, since MF synapses terminate on proximal dendrites close to the PC soma, their rapid rise time and large amplitude will quickly bring the membrane potential above threshold, generating action potentials that are temporally precise and largely independent of inhibition. We predict that rather than control the temporal precision of individual action potentials, the primary role of feedforward inhibition in the CA3 region of the hippocampus is to prevent run away excitation, the generation of plateau potentials, and repetitive burst firing within CA3 PCs by providing a robust GABAergic conductance. Of particular interest, CA3 PCs express L-type calcium channels in their soma and proximal dendrites near mossy fiber inputs (Westenbroek et al., 1990; Glazewski et al., 1993; Hell et al., 1993; Elliott et al., 1995). Developing CA3 pyramidal cells are predisposed to burst firing, which arises largely through activation of L-type calcium channels (Traub and Wong, 1982; Miles and Wong, 1983; Buzsaki, 1986). Although the role for single versus burst firing action potential generation in CA3 PCs is unclear, we have recently demonstrated that burst firing in CA3 PCs is directly linked to induction of long-term depression and the maturation of MF-excitatory synaptic transmission during the first 2-3 postnatal weeks (Lei et al., 2003; Ho et al., 2007, 2009). Burst firing in juvenile CA3 PCs triggers a switch from synapses primarily comprised of $\mathrm{Ca}^{2+}$-permeable, GluR2-lacking AMPA receptors to ones containing $\mathrm{Ca}^{2+} \mathrm{Ca}$-impermeable GluR2-containing AMPA receptors (Ho et al. 2009). It is possible that feedforward inhibitory control of CA3 burst firing provides an essential regulatory mechanism over mossy fiber maturation during development as well as acting as a gate for bursting in more mature networks.

In conclusion our data suggest that unlike other canonical feedforward inhibitory pathways in hippocampus and cortex, mossy fiber-driven feedforward inhibition does not substantially influence the timing of single action potentials CA3 PCs but rather provides a potent mechanism to prevent excessive depolarization and burst firing of CA3 PCs.

\section{References}

Acsády L, Kamondi A, Sík A, Freund T, Buzsáki G (1998) GABAergic cells are the major postsynaptic targets of mossy fibers in the rat hippocampus. J Neurosci 18:3386-3403.

Banke TG, McBain CJ (2006) GABAergic input onto CA3 hippocampal interneurons remains shunting throughout development. J Neurosci 26:11720-11725.

Buzsáki G (1986) Hippocampal sharp waves: their origin and significance. Brain Res 398:242-252.

Daw MI, Ashby MC, Isaac JT (2007) Coordinated developmental recruitment of latent fast spiking interneurons in layer IV barrel cortex. Nat Neurosci 10:453-461.

Elliott EM, Malouf AT, Catterall WA (1995) Role of calcium channel subtypes in calcium transients in hippocampal CA3 neurons. J Neurosci 15:6433-6444. 
Gabernet L, Jadhav SP, Feldman DE, Carandini M, Scanziani M (2005) Somatosensory integration controlled by dynamic thalamocortical feedforward inhibition. Neuron 48:315-327.

Głazewski S, Skangiel-Kramska J, Kossut M (1993) Development of NMDA receptor-channel complex and L-type calcium channels in mouse hippocampus. J Neurosci Res 35:199-206.

Glickfeld LL, Scanziani M (2006) Distinct timing in the activity of cannabinoid-sensitive and cannabinoid-insensitive basket cells. Nat Neurosci 9:807-815.

Glickfeld LL, Atallah BV, Scanziani M (2008) Complementary modulation of somatic inhibition by opioids and cannabinoids. J Neurosci 28:1824-1832.

Hell JW, Westenbroek RE, Warner C, Ahlijanian MK, Prystay W, Gilbert MM, Snutch TP, Catterall WA (1993) Identification and differential subcellular localization of the neuronal class C and class D L-type calcium channel alpha 1 subunits. J Cell Biol 123:949-962.

Henze DA, Wittner L, Buzsáki G (2002) Single granule cells reliably discharge targets in the hippocampal CA3 network in vivo. Nat Neurosci 5:790-795.

Higley MJ, Contreras D (2006) Balanced excitation and inhibition determine spike timing during frequency adaptation. J Neurosci 26:448-457.

Ho MT, Pelkey KA, Topolnik L, Petralia RS, Takamiya K, Xia J, Huganir RL, Lacaille JC, McBain CJ (2007) Developmental expression of $\mathrm{Ca}^{2+}$. permeable AMPA receptors underlies depolarization-induced long-term depression at mossy fiber CA3 pyramid synapses. J Neurosci 27:11651-11662.

Ho MT, Ho TM, Pelkey KA, Pelletier JG, Huganir RL, Lacaille JC, McBain CJ (2009) Burst firing induces postsynaptic LTD at developing mossy fibreCA3 pyramid synapses. J Physiol 587:4441-4454.

Jonas P, Major G, Sakmann B (1993) Quantal components of unitary EPSCs at the mossy fibre synapse on CA3 pyramidal cells of rat hippocampus. J Physiol 472:615-663.

Jung MW, McNaughton BL (1993) Spatial selectivity of unit activity in the hippocampal granular layer. Hippocampus 3:165-182.

Kamiya H, Shinozaki H, Yamamoto C (1996) Activation of metabotropic glutamate receptor type 2/3 suppresses transmission at rat hippocampal mossy fibre synapses. J Physiol 493:447-455.

Kwon HB, Castillo PE (2008) Role of glutamate autoreceptors at hippocampal mossy fiber synapses. Neuron 60:1082-1094.

Lawrence JJ, Grinspan ZM, McBain CJ (2004) Quantal transmission at mossy fibre targets in the CA3 region of the rat hippocampus. J Physiol 554:175-193.

Lei S, Pelkey KA, Topolnik L, Congar P, Lacaille JC, McBain CJ (2003) Depolarization-induced long-term depression at hippocampal mossy fiber-CA3 pyramidal neuron synapses. J Neurosci 23:9786-9795.
Losonczy A, Biro AA, Nusser Z (2004) Persistently active cannabinoid receptors mute a subpopulation of hippocampal interneurons. Proc Natl Acad Sci U S A 101:1362-1367.

Luna VM, Schoppa NE (2008) GABAergic circuits control input-spike coupling in the piriform cortex. J Neurosci 28:8851-8859.

Miles R, Wong RK (1983) Single neurones can initiate synchronized population discharge in the hippocampus. Nature 306:371-373.

Miles R, Toth K, Gulyás AI, Hájos N, Freund TF (1996) Differences between somatic and dendritic inhibition in the hippocampus. Neuron 16:815-823.

Mori M, Abegg MH, Gähwiler BH, Gerber U (2004) A frequencydependent switch from inhibition to excitation in a hippocampal unitary circuit. Nature 431(7007):453-456.

Nakashiba T, Young JZ, McHugh TJ, Buhl DL, Tonegawa S (2008) Transgenic inhibition of synaptic transmission reveals role of CA3 output in hippocampal learning. Science 319:1260-1264.

Pelkey KA, Lavezzari G, Racca C, Roche KW, McBain CJ (2005) mGluR7 is a metaplastic switch controlling bidirectional plasticity of feedforward inhibition. Neuron 46:89-102.

Pouille F, Scanziani M (2001) Enforcement of temporal fidelity in pyramidal cells by somatic feed-forward inhibition. Science 293:1159-1163.

Salin PA, Scanziani M, Malenka RC, Nicoll RA (1996) Distinct short-term plasticity at two excitatory synapses in the hippocampus. Proc Natl Acad Sci U S A 93:13304-13309.

Szabadics J, Soltesz I (2009) Functional specificity of mossy fiber innervation of GABAergic cells in the hippocampus. J Neurosci 29:4239-4251.

Toth K, McBain CJ (1998) Afferent-specific innervation of two distinct AMPA receptor subtypes on single hippocampal interneurons. Nat Neurosci 1:572-578.

Toth K, Suares G, Lawrence JJ, Philips-Tansey E, McBain CJ (2000) Differential mechanisms of transmission at three types of mossy fiber synapse. J Neurosci 20:8279-8289.

Traub RD, Wong RK (1982) Cellular mechanism of neuronal synchronization in epilepsy. Science 216:745-747.

Westenbroek RE, Ahlijanian MK, Catterall WA (1990) Clustering of L-type $\mathrm{Ca}^{2+}$ channels at the base of major dendrites in hippocampal pyramidal neurons. Nature 347:281-284.

Williams SH, Johnston D (1991) Kinetic properties of two anatomically distinct excitatory synapses in hippocampal CA3 pyramidal neurons. J Neurophysiol 66:1010-1020.

Yoshino M, Sawada S, Yamamoto C, Kamiya H (1996) A metabotropic glutamate receptor agonist DCG-IV suppresses synaptic transmission at mossy fiber pathway of the guinea pig hippocampus. Neurosci Lett 207: $70-72$. 